\title{
Erratum to: Advanced Practice Pharmacists: a retrospective evaluation of the efficacy and cost of ClinicaL Pharmacist PractitionErs managing ambulatory Medicare patients in North Carolina (APPLE-NC)
}

Michèle M. Kislan ${ }^{1 *}$, Adam T. Bernstein ${ }^{2}$, Loretta R. Fearrington ${ }^{3}$ and Timothy J. Ives ${ }^{4,5}$

\section{Erratum}

Following the publication of this article [1] it was brought to our attention that there was an error in the 'Total cost' paragraph of the 'Results' section.

The paragraph currently reads: "The total cost of OPV, EDV, IA, and prescribed medications in the CPP-R cohort was $1.46 \%$ lower, as compared to the PCP-A cohort $(p=0.026)$ (Data not shown due to proprietary nature of data)."

This should instead read: "The total cost of OPV, EDV, IA, and prescribed medications in the CPP-R cohort was $119.6 \%$ lower, as compared to the PCP-A cohort $(p=0.026)$ (Data not shown due to proprietary nature of data)."

\footnotetext{
Author details

${ }^{1}$ Yakima Valley Memorial Hospital, Yakima, WA 98902, USA. ${ }^{2}$ Department of Pharmacy, University of North Carolina Health Care, Chapel Hill, NC 27514, USA. ${ }^{3}$ The North Carolina Translational \& Clinical Sciences Institute, University of North Carolina at Chapel Hill, Chapel Hill, NC 27599-7064, USA. ${ }^{4}$ Eshelman School of Pharmacy, University of North Carolina at Chapel Hill, Chapel Hill, NC 27599-7574, USA. ${ }^{5}$ Division of General Medicine and Clinical Epidemiology, School of Medicine, University of North Carolina at Chapel Hill, Chapel Hill, NC 27599-7110, USA.
}

Received: 17 November 2016 Accepted: 17 November 2016 Published online: 28 November 2016

\footnotetext{
Reference

1. Kislan MM, et al. Advanced Practice Pharmacists: a retrospective evaluation

of the efficacy and cost of ClinicaL Pharmacist PractitionErs managing ambulatory Medicare patients in North Carolina (APPLE-NC). BMC Health Serv Res. 2016;16:607. doi:10.1186/s12913-016-1851-2.
}

\footnotetext{
* Correspondence: MicheleKislan@yvmh.org

'Yakima Valley Memorial Hospital, Yakima, WA 98902, USA
} 